\title{
Extramedullary relapse of multiple myeloma defined as the highest risk group based on deregulated gene expression data
}

\author{
Sabina Sevcikova ${ }^{\mathrm{a}, \mathrm{b}}$, Helena Paszekovac, Lenka Besse ${ }^{\mathrm{a}, \mathrm{b}}$, Lenka Sedlarikova ${ }^{\mathrm{a}, \mathrm{b}}$, Veronika Kubaczkovaa ${ }^{\mathrm{a}}$, Martina Almasia, ${ }^{\mathrm{a} b}$, \\ Ludek Pour ${ }^{\mathrm{d}}$, Roman Hajek ${ }^{\mathrm{a}, \mathrm{b}, \mathrm{e}}$
}

Background. Multiple myeloma (MM) is characterized by malignant proliferation of plasma cells (PC) which accumulate in the bone marrow (BM). The advent of new drugs has changed the course of the disease from incurable to treatable, but most patients eventually relapse. One group of MM patients (10-15\%) is considered high-risk because they relapse within 24 months. Recently, extramedullary relapse of MM (EM) has been observed more frequently. Due to its aggressivity and shorter survival, EM is also considered high-risk.

Aims. The goal of this study was to determine if the so-called high-risk genes published by the University of Arkansas group (UAMS) are even more deregulated in EM patients than in high-risk MM patients and if these patients may be considered high-risk.

Methods. Nine samples of bone marrow plasma cells from MM patients as well as 9 tumors and 9 bone marrow plasma cells from EM patients were used. Quantitative real-time PCR was used for evaluation of expression of 15 genes connected to the high-risk signature of MM patients.

Results. Comparison of high-risk plasma cells vs extramedullary plasma cells revealed 4 significantly deregulated genes (CKS1B, CTBS, NADK, YWHAZ); moreover, comparison of extramedullary plasma cells vs extramedullary tumors revealed significant differences in 9 out of 15 genes. Of these, 6 showed significant changes as described by the UAMS group (ASPM, SLC19A1, NADK, TBRG4, TMPO and LARS2).

Conclusions. Our data suggest that increasing genetic abnormalities as described by the gene expression data are associated with increased risk for EM relapse.

Key words: multiple myeloma, gene expression, high-risk disease, extramedullary relapse, qPCR

Received: November 7, 2014; Accepted with revision: March 31, 2015; Available online: April 15, 2015 http://dx.doi.org/10.5507/bp.2015.014

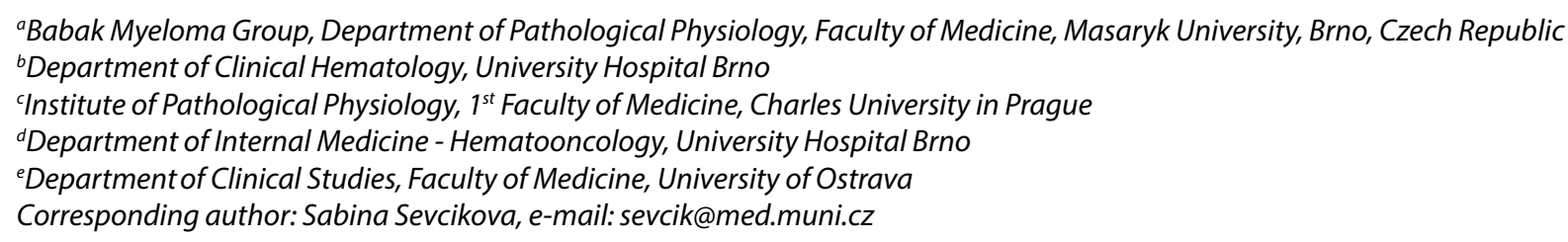

\section{INTRODUCTION}

Multiple myeloma (MM) is the second most common hematological malignancy in the world. It is characterized by malignant proliferation of a clone of plasma cells (PC) that produce a specific type of monoclonal immunoglobulin. Unfortunately, the cause of this disease is still unknown ${ }^{1}$. Clinical presentation and the development of the disease are very heterogeneous, from aggressive cases with multiple fractures of vertebrae and long bones, significant cytopenia and kidney damage to asymptomatic MM characterized by the presence of monoclonal immunoglobulin and increased amounts of PC in the bone marrow (BM) $\left(\right.$ ref. $\left.^{2}\right)$. Even though many advances in therapy have been made, MM is still considered an incurable disease since most patients eventually relapse ${ }^{3}$.

Based on the newest guidelines of the International Myeloma Working Group (IMWG) published in 2014, high-risk MM is defined by ISS stage II or III, the presence of either $\mathrm{t}(4,14)$ or del17p13 and comprises about $20 \%$ of patients ${ }^{4}$. These patients relapse early, their re- missions are of short duration and the disease eventually becomes refractory. Several attempts to identify these patients have been made, from FISH analyses to gene expression profiling (GEP). So far, several GEP profiles of high-risk MM have been published ${ }^{5-8}$. The GEP profile originating in the University of Arkansas (UAMS) was the first published validated model ${ }^{5}$. This model was based on the hypothesis that a group of genes are connected to shorter patient survival and can be used as an independent prognostic marker. The UAMS described a panel of 70 genes with either increased or decreased expression in the high-risk group of patients. An interesting observation was the position of many genes connected to high-risk signature on chromosome 1 (almost $50 \%$ of the 19 genes with decreased expression and $30 \%$ of 51 genes with decreased expression). As previously published, cytogenetic abnormalities, especially amplification of the long arm of chromosome 1, are connected to poor outcome for these patients. Several studies suggested that the percentage of cells with amplification of the 1q21 region is connected to disease progression ${ }^{9}$. Next to this, high-risk patients 
show high levels of beta- 2 microglobulin and creatinine, deletion of chromosome 13 and other abnormalities with negative influence on patient survival. This GEP model was later simplified to 17 gene expression out of the original 70 that still predict high-risk disease with $97.7 \%$ sensitivity 5 .

Extramedullary relapse of MM (EM) is defined by tumor cells found outside of the BM (ref. ${ }^{3}$ ). It is proposed that EM is caused by secondary mutations in PC subclones that cause unresponsiveness of these cells to the microenvironment of BM, and these PC then leave the $\mathrm{BM}$ (ref. ${ }^{10}$ ). The above mentioned adverse factors lead to resistance to treatment ${ }^{11,12}$. Clinically, three types of extramedullary lesions can be described: a) tumor mass adjacent to the bone and extending into soft tissues, b) soft tissue or visceral tumor that is not connected to the bone or c) diffuse infiltration of organs by PC without any obvious focal lesion ${ }^{3}$. Pour et al noted a marked decrease in survival for EM patients in comparison to MM patients (109 vs 38 months). Moreover, in EM patients, there was a difference between survival of patients with EM lesions infiltrating into soft organs in comparison to bone-related EM (30 vs 45 months). EM can be present either at the time of initial MM diagnosis (primary EM) or at the time of MM relapse (secondary EM) $\left(\right.$ ref. $\left.^{3}\right)$.

Primary EM is diagnosed in $4-16 \%$ of patients at the time of MM diagnosis. Long-term survival of these patients is significantly decreased when treated by standard therapy ${ }^{13,14}$. EM relapse was detected in $10-15 \%$ of patients when high-dose chemotherapy and transplant were used. Total survival was significantly shorter than for other forms of MM relapse ${ }^{15}$. The era of new drugs, such as immunomodulatory drugs and proteasome inhibitors, has increased survival of MM patients but reports of increased incidence of EM are being published ${ }^{3,16}$. As of now, it is still unclear if the reported incidence is true or if it is connected to better imaging methods, such as PET/CT $\left(\right.$ ref. $^{16}$ ).

In this study, we used expression of genes described in the UAMS GEP model to compare high-risk MM patients to EM patients. The aim was to test if these gene expression changes are more profound in EM patients, and are perhaps connected to the aggressiveness of EM relapse and the ability of EM PC to travel outside the BM.

\section{MATERIAL AND METHODS}

\section{Patients and sample preparation}

Retrospective samples from 9 high-risk MM patients at the time of diagnosis and 9 EM patients at the time of EM diagnosis from the Faculty Hospital Brno, Czech Republic, were included in this study. These high-risk MM patients relapsed within 2 years from diagnosis and thus were clinically defined as high-risk patients. All EM patients were diagnosed with EM relapse at the time of relapse of MM. For EM patients, BM and tumor samples were obtained. For MM patients, only BM samples were used. All patients signed the informed consent form approved by the Ethics committee of the hospital before entering the study. Clinical characteristics of the patients are described in Table 1.

\section{Plasma cell separation}

$\mathrm{BM}$ PC in the mononuclear cell fraction were enriched by anti-CD138+ immunomagnetic beads and sorted using AutoMACS (Miltenyi Biotec) as previously described ${ }^{17}$. Samples with $>80 \%$ purity of PC and frozen tissues at $-80{ }^{\circ} \mathrm{C}$ were used for RNA extraction.

\section{RNA extraction}

Total RNA was isolated from frozen PC using RNeasy ${ }^{\circledR}$ Mini Kit (Qiagen). RNA with purity ratio 260/280 > 1.80 (measured by Nanodrop ND-1000 spectrophotometer, Fisher Thermo Scientific, USA) was used for further analyses. High Pure RNA Tissue Kit (Roche, Basel, Switzerland) was used for the isolation of RNA from EM tissue. Samples weighing between 10 to $25 \mathrm{mg}$ were homogenized, and isolation of RNA was performed according to the manufacturers' protocol.

\section{Quantitative real-time PCR (qRT-PCR)}

Input of $100 \mathrm{ng}$ of total RNA was reverse transcribed using High Capacity cDNA Reverse Transcription Kit (Life Technologies, CA, USA). The obtained cDNA was preamplified with TaqMan ${ }^{\circledR}$ PreAmp Master Mix (Life Technologies, CA, USA) based on manufacturer instructions. qRT-PCR was performed using 7500 Real Time PCR System (Life Technologies, CA, USA) and TaqMan ${ }^{\circledR}$ Gene Expression Master Mix (Life Technologies, CA, USA). Experiments were performed in duplicates, and

Table 1. Patients' characteristics.

\begin{tabular}{lcc}
\hline & HR & EM \\
\hline Number of patients & 9 & 9 \\
Gender: males/females & $5 / 4$ & $7 / 2$ \\
Age at diagnosis (range) [years] & $58(47-81)$ & $65(52-79)$ \\
ISS stage: I-II-III [\%] & $55.6-22.2-22.2$ & $22.2-33.3-44.4$ \\
Durie-Salmon stadium: I-II-III [\%] & $0-0-100$ & $0-11-89$ \\
Ig isotype: IgG-IgA-IgD-light chains only-non-secretory [\%] & $50-25-0-12.5-12.5$ & $57-0-14-28-0$ \\
M-Ig (range) [g/L] & $24.4(0-76.7)$ & $20.9(0-49.2)$ \\
\hline Plasma cell infiltration in bone marrow (range) [\%] & $38.2(0-97)$ & $33.5(0-65)$ \\
\hline
\end{tabular}

HR- high-risk MM patients; EM- extramedullary relapse of MM 
commercially available human BM total RNA (cat no 636591, Clontech) was used as the calibrator for $2^{-\Delta \Delta \mathrm{Ct}}$ relative quantification approach.

The following genes were tested: $Y W H A Z, K I F 20 B$, NADK, LARS2, TBRG4, CKS1B, AIM2, ASPM, AHCYL1, CTBS, CLCC1, LTBP1, TMPO, SLC19A1, CCNA2, CCNB1, $K I F 14$. Expression of each gene was normalized to expression of endogenous housekeeping control gene, GAPDH (probe catalogue numbers are all located in Table 2).

Analysis of the output data was performed using 7500 SDS software (version 1.41, Life Technologies, CA, USA) using relative quantification approach.

\section{Statistical analysis}

Statistical significance of differences in continuous variables among groups of patients was analyzed using nonparametric Kruskal-Wallis or Mann-Whitney U test. Data were statistically analyzed with IBM SPSS Statistics, v.21. Differences with $P$-values $\leq 0.05$ were considered statistically significant.

\section{RESULTS}

\section{Comparison of normalized expression of selected genes in high-risk patients}

Statistically significant differences were established among three groups of patients - high-risk MM (HR PC) versus EM (EM PC) (Table 3a), HR PC versus EM tumor (EM T) (Table 3b) and EM PC versus EM T (Table 3c).

In the first comparison of gene expression in the HR PC versus EM PC groups, significant difference was determined only in 4 genes (CKSIB, CTBS, NADK, YWHAZ). Only $C T B S$ showed expression change in the expected direction - decrease in median from 2.09 in $\mathrm{HR}$ PC to 0.475 in EM PC $(P=0.009)$.

In the second comparison, we determined 4 genes with significant expression differences between HR PC and EM T groups. More specifically, ASPM and SLC19AI showed expression change in the expected direction - increase in median from 0.99 in HR MM to 6.9 in EM T for $\operatorname{ASPM}(P=0.008)$ and from 1.67 in HR MM to 7.55 in EM T for $S L C 19 A 1(P=0.005)$.

The third comparison of gene expression between EM PC and EM T groups revealed the greatest proportion of statistically significant differences in 9 out of 15 genes. Out of these 9 genes, 6 showed expression change in the expected direction - expression of ASPM (increase from 0.82 in EM PC to 6.9 in EM T; $P=0.006)$ and $S L C 19 A 1$ (increase from 2.095 in EM PC to 7.55 in EM T; $P=$ 0.006 ), then NADK, TBRG4, TMPO and LARS2 were observed. The expression of $N A D K$ gene increased from 1.34 in EM PC to 10.07 in EM T $(P=0.011)$, expression of TBRG4 increased from 2.22 in EM PC to 5.39 in EM T $(P=0.045)$. Expression of TMPO increased from 0.335 in EM PC to 1.18 in EM T $(P=0.045)$. Further, LARS2 expression was increased in EM T (from 1.2 in EM PC to 5.2 in EM T; $P=0.028$ ).

\section{DISCUSSION}

We used the expression of individual genes published in the UAMS GEP profile to check if their expression is even more deregulated in EM patients than in high-risk patients and if EM disease may be considered ultra highrisk disease.

We found several genes that significantly differ among compared groups. The CTBS gene is located on locus $1 \mathrm{p} 22$ and encodes lysosomal glycosidase involved in the degradation of oligosaccharides in glycoproteins ${ }^{18}$. We found no source that would link this gene or protein with MM or any another cancer. However, change in energy metabolism regulation appears to be one of the hallmarks of cancer ${ }^{19}$. Thus, it is possible that this gene is involved in MM pathogenesis in a general way.

The $A S P M$ gene (locus 1q31) was previously described as one of the genes that encode proteins involved in the cell cycle and mitosis; its expression is increased in the population of cells that are considered initial tumor cells in $\mathrm{MM}\left(\right.$ ref. $^{20}$ ). This paper showed that bortezomib treatment can reduce $\mathrm{MM}$ tumorigenicity by reduction of target gene expression, including $A S P M$, in this cell population. The increased expression that we found may play a role in tumor progression and be considered a marker of negative prognosis.

The SLC19A1 gene is located on chromosome 21 (locus 21q22.3), and its product is a membrane protein involved in the transport of folate and regulation of intracellular folate concentrations. Changes in folate metabolism caused by polymorphism in the SLC19A1 gene tends

Table 2. List of probes (Applied Biosystems) used in the experiments.

\begin{tabular}{ll}
\hline Gene & Probe number \\
\hline YWHAZ & Hs00237047_m1 \\
KIF20B & Hs01027505_m1 \\
NADK & Hs00224899_m1 \\
LARS2 & Hs01118920_m1 \\
TBRG4 & Hs00229429_m1 \\
$C K S 1 B$ & Hs02518862_g1 \\
AIM2 & Hs00175457_m1 \\
$A S P M$ & Hs00996458_m1 \\
AHCYL1 & Hs00198312_m1 \\
$C T B S$ & Hs00189273_m1 \\
$C L C C 1$ & Hs00209426_m1 \\
$L T B P 1$ & Hs00386448_m1 \\
$T M P O$ & Hs00162842_m1 \\
SLC19A1 & Hs00953344_m1 \\
$C C N A 2$ & Hs00153138_m1 \\
$C C N B 1$ & Hs00259126_m1 \\
KIF14 & Hs00978235_m1 \\
GAPDH & 4333764F
\end{tabular}


Table 3a. Comparison of gene expression between HR PC and EM PC groups.

\begin{tabular}{lccc}
\hline & HR PC (median - range) & EM PC (median - range) & $\boldsymbol{P}$ \\
\hline AIM2 & $29.22(3.44-231.58)$ & $24.44(8.34-85.66)$ & 0.697 \\
AMCYL1 & $0.95(0.49-7.84)$ & $0.875(0.50-1.31)$ & 0.259 \\
ASPM & $0.99(0.23-8.19)$ & $0.82(0.55-1.56)$ & 0.815 \\
CLCC1 & $3.94(1.31-16.12)$ & $4.145(2.94-7.87)$ & 0.755 \\
CKSIB & $46.61(5.5-75706.7)$ & $2.04(0.71-6.29)$ & $\mathbf{0 . 0 0 1}$ \\
CTBS & $2.09(0.52-11.56)$ & $0.475(0.26-1.08)$ & $\mathbf{0 . 0 0 9}$ \\
KIF14 & $1.19(0.3-19.8)$ & $0.93(0.0-7.4)$ & 0.640 \\
NADK & $8.85(2.39-71.3)$ & $1.34(0.64-4.69)$ & $\mathbf{0 . 0 0 1}$ \\
LTBP1 & $0.9(0.09-2.29)$ & $0.345(0.13-16.6)$ & 0.586 \\
TBRG4 & $2.82(1.24-16.44)$ & $2.22(1.28-3.94)$ & 0.276 \\
TMPO & $1.15(0.16-7.64)$ & $0.335(0.24-1.56)$ & 0.102 \\
SLC19A1 & $1.67(0.16-10.9)$ & $2.095(1.18-2.32)$ & 0.938 \\
KIF20B & $0.98(0.43-6.64)$ & $0.42(0.17-1.37)$ & 0.052 \\
LARS2 & $5.21(0.42-12.34)$ & $1.2(0.4-3.58)$ & 0.094 \\
YWHAZ & $4.16(1.81-13.19)$ & $2.245(1.65-4.75)$ & $\mathbf{0 . 0 4 3}$ \\
\hline
\end{tabular}

HR PC- high-risk MM- plasma cells; EM PC- extramedullary relapse- plasma cells

Table 3b. Comparison of gene expression between HR PC and EM T groups.

\begin{tabular}{lccc}
\hline & HR PC (median - range) & EM T (median - range) & $P$ \\
\hline AIM2 & $29.22(3.44-231.58)$ & $21.82(10.07-31.81)$ & 0.513 \\
AMCYL1 & $0.95(0.49-7.84)$ & $2.24(0.83-6.52)$ & 0.176 \\
ASPM & $0.99(0.23-8.19)$ & $6.9(1.93-9.05)$ & $\mathbf{0 . 0 0 8}$ \\
CLCC1 & $3.94(1.31-16.12)$ & $3.2(2.5-5.15)$ & 0.570 \\
CKS1B & $46.61(5.5-75706.7)$ & $7.86(0.76-14.74)$ & $\mathbf{0 . 0 1 0}$ \\
CTBS & $2.09(0.52-11.56)$ & $1.71(0.86-3.22)$ & 0.965 \\
KIF14 & $1.19(0.3-19.8)$ & $0.13(0.0-110.1)$ & 0.407 \\
NADK & $8.85(2.39-71.3)$ & $10.07(2.56-11.22)$ & 0.896 \\
LTBP1 & $0.9(0.09-2.29)$ & $0.9(0.14-1.91)$ & 0.930 \\
TBRG4 & $2.82(1.24-16.44)$ & $5.39(2.22-8.96)$ & 0.359 \\
TMPO & $1.15(0.16-7.64)$ & $1.18(0.59-4.25)$ & 0.727 \\
SLC19A1 & $1.67(0.16-10.9)$ & $7.55(5.55-16.82)$ & $\mathbf{0 . 0 0 5}$ \\
KIF20B & $0.98(0.43-6.64)$ & $0.93(0.3-1.88)$ & 0.541 \\
LARS2 & $5.21(0.42-12.34)$ & $5.2(1.33-10.99)$ & 0.150 \\
YWHAZ & $4.16(1.81-13.19)$ & $1.16(0.42-1.50)$ & $\mathbf{0 . 0 0 1}$ \\
\hline
\end{tabular}

HR PC- high-risk MM- plasma cells; EM T- extramedullary relapse - tumor tissue

Table 3c. Comparison of gene expression between EM PC and EM T groups.

\begin{tabular}{lccc}
\hline & EM PC (median - range) & EM T (median - range) & $\boldsymbol{P}$ \\
\hline AIM2 & $24.44(8.34-85.66)$ & $21.82(10.07-31.81)$ & 0.855 \\
AMCYL1 & $0.875(0.50-1.31)$ & $2.24(0.83-6.52)$ & $\mathbf{0 . 0 2 8}$ \\
ASPM & $0.82(0.55-1.56)$ & $6.9(1.93-9.05)$ & $\mathbf{0 . 0 0 6}$ \\
CLCC1 & $4.145(2.94-7.87)$ & $3.2(2.5-5.15)$ & 0.465 \\
CKS1B & $2.04(0.71-6.29)$ & $7.86(0.76-14.74)$ & 0.144 \\
CTBS & $0.475(0.26-1.08)$ & $1.71(0.86-3.22)$ & $\mathbf{0 . 0 1 1}$ \\
KIF14 & $0.93(0.0-7.4)$ & $0.13(0.0-110.1)$ & 1.000 \\
NADK & $1.34(0.64-4.69)$ & $10.07(2.56-11.22)$ & $\mathbf{0 . 0 1 1}$ \\
LTBP1 & $0.345(0.13-16.6)$ & $0.9(0.14-1.91)$ & 0.584 \\
TBRG4 & $2.22(1.28-3.94)$ & $5.39(2.22-8.96)$ & $\mathbf{0 . 0 4 5}$ \\
TMPO & $0.335(0.24-1.56)$ & $1.18(0.59-4.25)$ & $\mathbf{0 . 0 4 5}$ \\
SLC19A1 & $2.095(1.18-2.32)$ & $7.55(5.55-16.82)$ & $\mathbf{0 . 0 0 6}$ \\
KIF20B & $0.42(0.17-1.37)$ & $0.93(0.3-1.88)$ & 0.201 \\
LARS2 & $1.2(0.4-3.58)$ & $5.2(1.33-10.99)$ & $\mathbf{0 . 0 2 8}$ \\
YWHAZ & $2.245(1.65-4.75)$ & $1.16(0.42-1.50)$ & $\mathbf{0 . 0 0 6}$ \\
\hline
\end{tabular}

EM PC- extramedullary plasma cells; EM T- extramedullary tumor tissue

The lines represent individual gene expression and columns patient groups (HR PC - high-risk, EM PC- EM plasma cells and EM T - EM tumor groups). The values in the first two columns show median of gene expression and range (minimum-maximum). When the difference was statistically significant $(\leq 0.05)$, the value is in bold. 
to slow down MM progression because tumor cells make great demands on folate supply in order to maintain DNA synthesis at high levels ${ }^{21}$. For this reason, we can expect that increased SLC19A1 expression will accelerate disease progression, reflecting its inclusion in the high-risk gene panel. These findings correspond to our assumption that EM T represents a more aggressive type of $\mathrm{MM}$ with a greater rate of genomic abnormalities.

The NADK gene (locus 1p36.21) encodes NAD kinase that catalyzes the transfer of a phosphate group from ATP to NAD to generate NADP+, which is an electron donor for all biosynthetic reactions ${ }^{22}$. Inhibition of NAD kinase enhances the degradation of the dihydrofolate reductase (DHFR), which plays a key role in DNA synthesis and for many diseases, including cancer; it represents a therapeutic target ${ }^{23}$. Degradation of DHFR is then associated with inhibition of tumor cell growth. For this reason, inhibition of NAD kinase is considered a promising target for cancer treatment. Increased expression of the $N A D K$ gene found in this study may constitute one of the steps in $\mathrm{MM}$ progression.

The TBRG4 gene is located on 7p14-p13 and encodes a regulator of TGF- $\beta$ (Transforming Growth Factor- $\beta$ ), which is of particular importance for MM in regulating hematopoiesis ${ }^{24}$. Increased levels of TGF- $\beta$ in the BM microenvironment is caused by its simultaneous production by both MM and stromal cells and it induces an increase of IL- 6 and VEGF secretion, major cytokines involved in tumor cell proliferation and angiogenesis. On the other hand, TGF- $\beta$ inhibits proliferation and immunoglobulin secretion of normal B-cells. Therefore, it is clear that regulation of TGF- $\beta$ plays an important role in MM development and progression ${ }^{25}$.

The TMPO gene is located on locus 12q22. Agnelli et al uncovered critical genes associated with poor prognosis by compilation of gene regulatory networks from expression data of $1883 \mathrm{MM}$ patients, and one of them was TMPO (ref. ${ }^{26}$ ). This gene is involved in the organization of the nuclear envelope structure and is deregulated in different types of hematological malignancies. Finding the same gene in another prognostic model reflects its importance; its increasing expression in EM T patients is consistent with our assumption.

LARS2 encodes mitochondrial leucyl-tRNA synthetase 2, an enzyme important in translation. The only malignant disease with known connection to this gene is nasopharyngeal carcinoma; however, in this case, it is associated with decreased expression due to frequent deletion of locus $3 \mathrm{p} 21.3$, and even the authors of this study found no other source of evidence of its role in tumorigenesis in the literature ${ }^{27}$.

Based on our data, it seems that there are different clones of PC in the BM of EM patients when compared to the tumors. The tumors are more aggressive because the development outside of BM requires further accumulation of genetic changes. This fact is confirmed by observed expression increase of six genes; out of which four ( $A S P M$, SLC19A1, TBRG4 and TMPO) have a direct connection to MM as proven by other studies $21,26,28,29$.
Differences in gene expression between high-risk patient groups and associated MM heterogeneity, e.g. in the clinical manifestation of the disease, survival and response to chosen therapy, are one of the major complications in creating predictive models based on GEP alone. Moreover, EM represents an aggressive and advanced state of malignant transformation; loss of BM microenvironment dependence in EM tumors indicates further genomic disruption. Comparison of the high-risk UAMS gene set with our high-risk patients showed that these four genes are significantly upregulated, which confirms the results obtained by UAMS. Moreover, these genes are even more upregulated in EM relapse of MM.

The highest risk group determined by the highest gene expression was EM tumors. This observation corresponds with the assumption that this type of MM representing a "clone" located outside the BM microenvironment had to accumulate more genomic abnormalities and thus its expression must be different from others. Based purely on expression of the chosen genes, our data suggest increasing risk of $\mathrm{MM}$ is associated with the accumulation of genetic abnormalities in the direction of HR PC < EM PC $<$ EM T. To confirm this conclusion, it would be necessary to perform whole-genome profiling for statistically significant differences between genes with confirmed links to $\mathrm{MM}$ or EM and their progression.

\section{ACKNOWLEDGEMENTS}

We would like to thank all the patients, their caregivers and our data-managers for allowing us to do this study. This work was supported by grants of Ministry of Health IGA NT12130 and NT14575 and a grant of the Ministry of Health, Czech Republic - conceptual development of research organization $(\mathrm{FNBr}, 65269705)$ and Masaryk University grant (MUNI/A/1549/2014).

We would also like to thank Bozena Hanakova, Barbora Sablikova and Marcela Stouracova for technical assistance. John B. Smith proofread this manuscript.

Author contribution: SS, HP: manuscript writing, performed experiments; SS: designed experiments; LB, LS, VK, MA: literature search, data analysis; LP, RH: collected patient samples, manuscript writting; RH: final revision.

Conflict of interest statement: The authors state that there are no conflicts of interest regarding the publication of this article.

\section{REFERENCES}

1. Hajek R, Adam Z, Scudla V, Maisnar V, Bacovsky J, Spicka I, Krejci M, Kessler P, Minarik J, Sandecka V, Radocha J, Flochova E, Gregora E, Gumulec J, Kafkova A, Kuglik P, Mistrik M, Palasthy S, Pavlicek P, Penka M, Pika T, Pour L, Racil Z, Rihova L, Smetana J, Schutzova M, Stecova N, Stefanikova Z, Straub J, Tothova E. Guidelines 2012: Diagnosis and treatment of multiple myeloma. Transfuze Hematol dnes 2012;18:1-89.

2. Anderson KC, Alsina $M$, Bensinger W, Biermann JS, Chanan-Khan A, Cohen AD, Devine S, Djulbegovic B, Faber EA Jr, Gasparetto 
C, Huff CA, Kassim A, Medeiros BC, Meredith R, Raje N, Schriber J, Singhal S, Somlo G, Stockerl-Goldstein K, Treon SP, Tricot G, Weber DM, Yahalom J, Yunus F, National Comprehensive Cancer Network. NCCN clinical practice guidelines in oncology: multiple myeloma. J Natl Compr Canc Netw 2011;9(10):1146-83.

3. Pour L, Sevcikova S, Greslikova H, Kupska R, Majkova P, Zahradova L, Sandecka V, Adam Z, Krejci M, Kuglik P, Hajek R. Soft-tissue extramedullary multiple myeloma prognosis is significantly worse in comparison to bone-related extramedullary relapse. Haematologica 2014;99(2):360-4.

4. Chng WJ, Dispenzieri A, Chim CS, Fonseca R, Goldschmidt $\mathrm{H}$, Lentzsch S, Munshi N, Palumbo A, Miguel JS, Sonneveld P, Cavo M, Usmani S,Durie BG, Avet-Loiseau H; International Myeloma Working Group. IMWG consensus on risk stratification in multiple myeloma. Leukemia 2014;28(2):269-77.

5. Shaughnessy JD Jr, Zhan F, Burington B, Huang Y, Colla S, Hanamura I, Stewart JP, Kordsmeier B, Randolph CH, Williams DR, Xiao Y, Xu H, Epstein J, Anaissie E, Krishna SG, Cottler-Fox M, Hollmig K, Mohiuddin A, Pineda-Roman M, Tricot G, van Rhee F, Sawyer J, Alsayed Y, Walker R, Zangari M, Crowley J, Barlogie B. A validated gene expression model of high-risk multiple myeloma is defined by deregulated expression of genes mapping to chromosome 1. Blood 2007;109(6):2276-84.

6. Decaux O, Lodé L, Magrangeas F, Charbonnel C, Gouraud W, Jézéque P, Attal M, Harousseau JL, Moreau P, Bataille R, Campion L, AvetLoiseau H, Minvielle S; Intergroupe Francophone du Myélome. Prediction of survival in multiple myeloma based on gene expression profiles reveals cell cycle and chromosomal instability signatures in high-risk patients and hyperdiploid signatures in low-risk patients: a study of the Intergroupe Francophone du Myélome. J Clin Oncol 2008;26(29):4798-805.

7. Moreaux J, Klein B, Bataille R, Descamps G, Maïga S, Hose $D$, Goldschmidt $H$, Jauch $A$, Rème $T$, Jourdan $M$, Amiot $M$, PellatDeceunynck $C$. A high-risk signature for patients with multiple myeloma established from the molecular classification of human myeloma cell lines. Haematologica 2011;96(4):574-82.

8. Kuiper R, Broyl $A$, de Knegt $Y$, van Vliet $M H$, van Beers $E H$, van der Holt B, el Jarari L, Mulligan G, Gregory W, Morgan G, Goldschmidt H, Lokhorst HM, van Duin M, Sonneveld P. A gene expression signature for high-risk multiple myeloma. Leukemia 2012;26(11):2406-13.

9. Hanamura I, Stewart JP, Huang Y, Zhan F, Santra M, Sawyer JR, Hollmig $K$, Zangarri $M$, Pineda-Roman $M$, van Rhee $F$, Cavallo $F$, Burington B, Crowley J, Tricot G, Barlogie B, Shaughnessy JD Jr. Frequent gain of chromosome band 1q21 in plasma-cell dyscrasias detected by fluorescence in situ hybridization: incidence increases from MGUS to relapsed myeloma and is related to prognosis and disease progression following tandem stem-cell transplantation. Blood 2006;108(5):1724-32.

10. Oriol A. Multiple myeloma with extramedullary disease. Adv Ther 2011;28 (7):1-6.

11. Katodritou E, Gastari V, Verrou E, Hadjiaggelidou C, Varthaliti M, Georgiadou S, Laschos K, Xirou P, Yiannaki E, Constantinou $\mathrm{N}$, Markala D, Zervas K. Extramedullary (EMP) relapse in unusual locations in multiple myeloma: Is there an association with precedent thalidomide administration and a correlation of special biological features with treatment and outcome? Leuk Res 2009;33(8):1137-40.

12. Anderson KC. New insights into therapeutic targets in myeloma. Hematology Am Soc Hematol Educ Program 2011;2011:184-90.

13. Wu P, Davies FE, Boyd K, Thomas K, Dines S, Saso RM, Potter $M N$, Ethell ME, Shaw BE, Morgan GJ. The impact of extramedullary disease at presentation on the outcome of myeloma. Leuk Lymphoma 2009;50(2):230-35.

14. Damaj G, Mohty M, Vey N, Dincan E, Bouabdallah R, Faucher C, Stoppa AM, Gastaut JA. Features of extramedullary and extraos- seous multiple myeloma: a report of 19 patients from a single center. Eur J Haematol 2004;73(6):402-6.

15. Terpos E, Rezvani K, Basu S, Milne AE, Rose PE, Scott GL, Rahemtulla A, Samson D, Apperley JF. Plasmocytoma relapses in the absence of systemic progression post-high-dose therapy for multiple myeloma. Eur J Haematol 2005;75(5):376-83.

16. Usmani SZ, Heuck C, Mitchell A, Szymonifka J, Nair B, Hoering A, Alsayed Y, Waheed S, Haider S, Restrepo A, Van Rhee F, Crowley $\mathrm{J}$, Barlogie B. Extramedullary disease portends poor prognosis in multiple myeloma and is over-represented in high-risk disease even in the era of novel agents. Haematologica 2012;97(11):1761-7.

17. Buresova I, Cumova J, Kovarova L, Stossova J, Dementyeva E, Kryukov F, Sevcikova S, Svachova H, Hajek R. Bone marrow plasma cell separation - validation of separation algorithm. Clin Chem Lab Med 2012;50(6):1139-40.

18. Aronson NN Jr, Kuranda MJ. Lysosomal degradation of Asn-linked glycoproteins. FASEB J 1989;3(14):2615-22.

19. Hanahan D, Weinberg RA. Hallmarks of cancer: the next generation. Cell 2011;144(5):646-74.

20. Nara $M$, Teshima $K$, Watanabe A, Ito $M$, Iwamoto $K$, Kitabayashi A, Kume M, Hatano Y, Takahashi N, lida S, Sawada K, Tagawa H. Bortezomib reduces the tumorigenicity of multiple myeloma via downregulation of upregulated targets in clonogenic side population cells. PLoS One 2013;8(3):e56954.

21. Maggini V, Buda G, Galimberti S, Conidi E, Guiliani N, Morabito F, Genestreti G, lacopino P, Rizzoli V, Barale R, Petrini M, Rossi AM. Association of folate transporter SLC19A1 polymorphisms with the outcome of multiple myeloma after chemotherapy and tandem autologous transplantation. Leukemia 2007;21(1):176-8.

22. Lerner F, Niere M, Ludwig A, Ziegler M. Structural and functional characterization of human NAD kinase. Biochem Biophys Res Commun 2001;288(1):69-74.

23. Hsieh YC, Tedeschi P, Adebisi Lawal R, Banerjee D, Scotto K, Kerrigan $J E$, Lee KC, Johnson-Farley N, Bertino JR, Abali EE. Enhanced degradation of dihydrofolate reductase through inhibition of NAD kinase by nicotinamide analogs. Mol Pharmacol 2013;83(2):339-53.

24. Urashima M, Ogata A, Chauhan D, Hatziyanni M, Vidriales MB, Dedera DA, Schlossman RL, Anderson KC. Transforming growth factor-beta1: differential effects on multiple myeloma versus normal B cells. Blood 1996;87(5):1928-38.

25. Kubiczkova L, Sedlarikova L, Hajek R, Sevcikova S. TGF- $\beta$ - an excellent servant but a bad master. J Transl Med 2012;10:183.

26. Agnelli L, Forcato M, Ferrari F, Tuana G, Todoerti K, Walker BA, Morgan GJ, Lombardi L, Bicciato S, Neri. The reconstruction of transcriptional network reveals critical genes with implications for clinical outcome of multiple myeloma. Clin Cancer Res 2011;17(23):7402-12.

27. Zhou W, Feng $X$, Li H, Wang L, Zhu B, Liu W, Zhao M, Yao K, Ren C. Inactivation of $L A R S 2$, located at the commonly deleted region 3 p21.3, by both epigenetic and genetic mechanisms in nasopharyngeal carcinoma. Acta Biochim Biophys Sin (Shanghai) 2009;41(1):5462.

28. Cohen Y, Gutwein O, Garach-Jehoshua O, Bar-Haim A, Kornberg A. The proliferation arrest of primary tumor cells out-of-niche is associated with widespread downregulation of mitotic and transcriptional genes. Hematology 2014;19(5):286-92.

29. Sarasquete ME, Martínez-López J, Chillón MC, Alcoceba M, Corchete LA, Paiva B, Puig N, Sebastián E, Jiménez C, Mateos MV, Oriol A, Rosiñol L, Palomera L, Teruel Al, González Y, Lahuerta JJ, Bladé J, Gutiérrez NC, Fernández-Redondo E, González M, San Miguel JF, García-Sanz R. Evaluating gene expression profiling by quantitative polymerase chain reaction to develop a clinically feasible test for outcome prediction in multiple myeloma. $\mathrm{Br} \mathrm{J}$ Haematol 2013;163(2):223-34. 